\title{
The role of Lactic Acid Bacteria in Gut Microbiota and Mucosal Immune System
}

\section{El papel de las bacterias del ácido láctico en la microbiota intestinal y el sistema inmunitario de la mucosa}

DOI: $10.46932 /$ sfjdv2n3-009

Received in: March 1st, 2021

Accepted in: May 30th, 2021

\author{
Siyu Lu \\ La Trobe University, Animal and Veterinary Biosciences, Plenty Rd \&, Kingsbury Dr, Bundoora VIC \\ 3086, Victoria, Australia \\ Corresponding author: lusiyu106@gmail.com
}

\begin{abstract}
In recent years, nutritionists and microbiologists have paid more attention to the utilization of dietary supplementation produced by natural food resources, to improve the immune system function of the human and animals. People are more willing to obtain nature supplementations due to the growing awareness of food safety, and the side effects of excessive consumption of manufactured supplements. Fermented food are introduced to provide beneficial effects on health because of the characteristics of $\mathrm{LAB}$, which are temporary integrative microorganisms in the intestinal microbiota. And they could not only balance the micro-ecosystem of the host by secreting microbiome, but also enhance the beneficial properties of autonomous microorganisms. This review aims to discuss the of LAB yogurt to the gut microbiota based on recent research.
\end{abstract}

\section{RESUMEN}

En los últimos años, los nutricionistas y microbiólogos han prestado más atención a la utilización de suplementos dietéticos producidos por recursos alimentarios naturales, para mejorar la función del sistema inmunitario del ser humano y de los animales. La gente está más dispuesta a obtener suplementos de la naturaleza debido a la creciente concienciación sobre la seguridad alimentaria y los efectos secundarios del consumo excesivo de suplementos manufacturados. Los alimentos fermentados se introducen para proporcionar efectos beneficiosos para la salud debido a las características de las BAL, que son microorganismos integradores temporales en la microbiota intestinal. Y no sólo podrían equilibrar el microecosistema del huésped mediante la secreción de microbioma, sino también potenciar las propiedades beneficiosas de los microorganismos autónomos. El objetivo de esta revisión es discutir la relación entre el yogur y las BAL con la microbiota intestinal, basándose en investigaciones recientes.

\section{INTRODUCTION}

In recent research, scientists elevated their interests in exploring the potential functions of natural resources as dietary supplements to maintain health, instead of manufactured supplements. Dietary supplementations have been reported with fewer side effects than manufactured supplements[1]. In 2018, Galior et al[1] researched on manufacturing errors of vitamin D, a series of side effects were caused due to intoxication and, including vomiting, low appetite, dehydration and pain[1]. However, natural 
resources, such as fermented food, are more likely to bring various of beneficial effects, such as detoxification, health promotion, and shelf-life extension[2].

Acidic fermentation is an established traditional method to produce fermented dairy products through milk acidification. Yogurt is one of the most common fermented products. It has been recommended as an alternative choice of milk for the estimated $75 \%$ of the population worldwide who have lactose intolerance to achieve dairy nutrition[3]. Live bacterial culture in yogurt could convert lactose into lactic acid, acetic acid, ethanol or carbon dioxide, hence remove lactose intolerance symptoms[4, 5]. Major lactic acid bacteria species in yogurt (LAB-Y) are Lactobacillus delbrueckii subsp. bulgaricus, Streptococcus themophlus, Bifidobacterium. These LAB-Y are probiotic, which secrete microbiome to regulate the micro-ecosystem of the host, preserve the homeostasis of intestinal microbiota, and promote the mucosal immune system

The unbalance between pathogenic microorganisms and beneficial microbiota might cause the dysbiosis of microbiota, which might lead to immune function disorder, infections, inflammation and autoimmunity susceptibility[7]. LAB-Y could help maintaining the homeostasis of gut microbiota by integrating the allochthonous bacteria to the residence of autonomous[6]. Also, LAB-Y modulate intestinal function, by eliminating the pathogenic microorganisms and preserving the homeostasis of beneficial microbiota[2].

In this review, the relationship between LAB-Y and gut microbiota will be discussed first. Then The relationship between the gut microbiota and immune system will be illustrated. Based on recent research, the contribution of LAB-Y in human and mice gut microbiota, and how LAB-Y promote the development in the mucosal immune system will be explained.

\section{LAB AND GUT MICROBIOTA}

\subsection{THE INHABITANT OF MICROORGANISM IN GUT MICROBIOTA}

Captions should be typed in 9-point Times. They should be centred above the tables and flush left beneath the figures. The gut microbiota located in the digestive system, is mostly abundant within the large intestine[8]. The gut microbiota is the community of up to 100 trillion microorganisms[2].The large number of microorganisms are belonged to five dominant phyla[9]. Firmicutes and Bacteroidetes are the major two bacterial phyla in the gut microbiota, which constitute about $90 \%$ of the total microbial communities[10]. While other subdominant phyla, Actinobacteria, Proteobacteria, Verrucomicrobia are the less abundant species[10].

\subsection{THE HOMEOSTASIS OF GUT MICROBIOTA (EUBIOSIS AND DYSBIOSIS)}


Both dominant and subdominant phyla have an irreplaceable contribution and symbiotic relationship to the homeostasis of the host[10]. Different genera present a variety of functions that assist the host. These functions include promoting the utilization of essential nutrients, metabolization of indigestible food, modulation of acquired immunity and innate aspects. The gut microbiome helps to prevent the pathogen from invading the host or cause immune disease.

Although Bifidobacterium, Lactobacillus presented with a small abundance in the gut microbiota, they significantly contribute to the eubiosis in the gut lumen, by converting lactate to butyrate or propionate, in order to prevent the accumulation of lactate, as well as to stabilize colonic microbiota[11]. Bifidobacterium belong to Actinobacteria phyla which produces short chain fatty acids (SCFA), including acetate, butyrate, propionate[12]. While Lactobacillus are from Firmicutes, they generate lactic acid as the final production[12].

However, the reduction of Bifidobacterium and Lactobacillus in gut microbiota might lead to the dysbiosis of intestinal immune system. Dysregulation of microorganisms may result in the host developing dysfunction[11]. Under the dysbiosis condition in gut microbiota, the composition of microorganisms alters, and more virulent microorganisms are selected instead of commensal beneficial ones [7]. The imbalance status would further trigger a series of deleterious effects on the host[13]. The host would become more susceptible to infections due to the reduction of antimicrobial peptides (AMPs), secretory $\operatorname{sg} \mathrm{A}(\operatorname{Ig} \mathrm{A})$ and mucins in mucosal barrier, accompanied with hypersensitivity, autoimmune disorder, or inflammatory diseases[13, 14].

\section{GUT MICROBIOTA AND IMMUNE SYSTEM}

A significant positive correlation coefficient was found between the gut microbiota and the mucosal immune system. The eubiosis in gut microbiota could better stimulate the activation of AMPs, sIgA and mucin, to protect the mucosal barrier against the invasion of exotic microorganisms. Differently, the dysbiosis of gut microbiota might lead to the dysregulation and dysfunction of mucosal immune system, with less secretion and activation of immune cells. More pathogenic microorganisms are likely to permeate intestinal mucus, and it could further affect the regular function of innate and adaptive immune system [7, 13, 14].

\section{LAB AND IMMUNE SYSTEM}

The mucosal immune system is the first immune defence barrier which eliminates more than $90 \%$ of pathogenic microorganisms and it is the largest immune organ in the body[15]. The integrity of mucosa is associated with the anti-inflammatory activity, immune defensive function in innate immunity and 
adaptive immunity[16]. Three distinct departments have to be maintained properly, in order to achieve a healthy mucosa, including the mucosal barrier, epithelial cell and immune cells in the lamina propria (LP)[15].

LAB plays an important role in maintaining the proper function in mucosal immune system, as well as the prevention of leaky epithelium and inflammatory syndrome, to avoid the increase permeability of pathogenic molecules into the bloodstream. [17, 18].

In the gut lumen, LAB provide butyrate for colonocytes to maintain mucosal integrity, generate lactic acid, bacteriocins, $\mathrm{H}_{2} \mathrm{O}_{2}$ or inhibitory agents to directly prohibit viruses before they adhere mucosal barrier, then guard the intestinal epithelial cell (IEC) receptors against the pathogens, to protect the integrity of epithelium[16].

The integrity of epithelium would therefore contain more anti-inflammatory agents, including mucin, sIgA, AMPs that can better protect the mucosal barrier from the invasion of microbial translocation[11]. Furthermore, IECs stimulate the production of BAFF and APRIL in activated B cell to increase sIgA, and signal immune-related cytokines and chemokines in the acquired immune system, to further inhibit the interaction between symbiotic microorganism and epithelium, including thymus stimulating protein, macrophages, T cells on antigen presenting cells, such as dendric cells (DC)[16, 19].

\section{YOGURT LAB STRAINS APPLICATIONS}

\subsection{HUMAN ASPECT}

In 2017, Lee et al.,[15] used dairy yogurt containing Lactobacillus. paracesei, Bifidobacterium. lactis compared to heat-treated Lactobacillus. Plantarum milk. In order to find out their differences in immunostimulatory effects. 200 healthy elderly were divided into two groups $(n=100)$. Their serum cytokine interleukin (IL)-12, immunoglobulin (Ig) concentration G1 and natural killer (NK) cells activity were measured, after $12.0 \times 108 \mathrm{CFU} /$ day consumption for twelve weeks[15].

IL12 is important to the activities of NK cells and T lymphocytes. It stimulates the production of IFN- $\gamma$ and TNF- $\alpha$ and differentiates into naïve T cells and Th1 cell[17]. IgG1is the major antibody in humoral immunity that contributes predominantly in the secondary immune response [20]. NK cells belong to innate lymphocytes group in the innate immune system, they are cytotoxic and contain granzymes, which induce apoptosis to eliminate harmful cells[21, 22].

The result showed that dairy yogurt group performed with a remarkable influence on enhancing IL-12, IgG1 concentration, NK cells activity, compared to the heat-treated milk group. 48 people who required antibiotics, experienced weight change more than 5\% from baseline, non-compliant white blood cell level at baseline, were excluded from the result[6]. 


\subsection{ANIMAL ASPECT}

In 2020, Lai et al discovered that different LAB strains could trigger diverse effects on immune responses[23]. They compare a novel strain, Bacillus sp. DU106 to three previously found LAB strains, Bifidobacterium lactis B94, Lactobacillus rhamnosus R11 and Lactobacillus acidophilus R418 (which are found to be beneficial to health)[23]. 50 similar condition mice were grouped into 5 different LAB treatments $(\mathrm{n}=10)$. After 30 days, their thymus cells, spleen cells indexes and the Tlymphocyte conversion rate were measured.

The thymus is the initial lymphoid organ for the development and cultivation of $\mathrm{T}$ and $\mathrm{B}$ lymphocytes. The spleen is the secondary lymphoid organ for lymphocytes to detect antigens and elevate immune response $[24,25]$. Therefore, the increase in thymus and spleen cell indexes indicate the potential growth of T and B cells, which could contribute to a higher stimulation of immune response.

$\mathrm{T}$ cells play an essential role in the adaptive immune system. They turn into $\mathrm{T}$ cells after the maturation in the thymus. The subsets of $\mathrm{T}$ cells have different distinct functions, such as the detection of antigenic cells, the stimulation of cytokines, motivation of macrophages [25]. Thus, the higher conversion rate of $\mathrm{T}$ cells s means more mature $\mathrm{T}$ cells and their subsets will be generated [24].

sIgA and sIgG are antibodies secreted by B cells, they bind to antigens and trap the pathologic cells from contacting with epithelial cells on luminal surface [26].

The result showed that different strains of LAB, even from the same genera, could contribute to the mucosal immune system differently (Table 1). In comparison, Bacillus sp. DU106 presented with a relatively higher activation and promotion on thymus and spleen cells index, the conversion rate of $\mathrm{T}$ lymphocyte, as well as the concentration of sIgA and sIgG. But Bifidobacterium lactis B94, Lactobacillus rhamnosus R11 and Lactobacillus acidophilus R418 also showed with a smaller contribution, compared to the control group (Table 1).

Table 1. Overview of secretory IgA and secretory $\operatorname{IgG}$ in five treatment groups.

\begin{tabular}{|l|l|l|}
\hline Group & sIgA & sIgG \\
\hline COD & 1.15 & 0.09 \\
\hline R11 & $1.15+0.35$ & 0.09 \\
\hline R418 & $1.15+0.10$ & 0.09 \\
\hline B94 & $1.15+0.45$ & $0.09+0.04$ \\
\hline DU106 & $1.15+0.75$ & $0.09+0.06$ \\
\hline
\end{tabular}

\section{CONCLUSION AND FUTURE PERSPECTIVES}

Both yogurt treatments on healthy elderly and mice research proved that the ingestion of yogurt would present beneficial effects on the regulation function in the mucosal immune system, including the 
increase of IL-12, the activity of NK cells, the number of thymus cells and spleen cells, the concentration of sIgA and sIgG. However, both researches only selected healthy human and mice to discover the contribution of LAB. People under different health conditions were excluded from the experiment, such as the antibiotics acquired, weight gained groups results. LAB by far could only be considered to have prophylactic function for healthy human and animals. Further researches are needed for observing the LAB functions on different groups of people and animals.

According to Redondo-Useros[6], LAB are allochthonous microorganisms, and they could only transiently integrate to the residential of gut microbiota. However, the continuous or regular ingestion of LAB might be needed, in order to obtain long-term health effect. The discontinuation of LAB ingestion might return the beneficial effects back to the baseline due to its transient characteristic [6]. Measurements of 'after treatment' groups are needed to be done, in order to discover the stability of LAB in gut microbiota, and its specific alterations in different aspects. In addition, Joukar et al [27] mentioned a better utilization to magnify the beneficial characteristics of LAB by inducing LAB for personalized cancer treatment in the future. Since more cancer cells might be eliminated due to the development of mucosal system triggered by LAB. Microbiota-target therapy could be developed by ligating allochthonous LAB strains to autochthonous microorganisms to enhance the activation of mucosal immune system.

\section{ACKNOWLEDGMENT}

I would like to thank Danyi Cheng for providing advices and correcting the grammar mistakes of this literature review. 


\section{REFERENCES}

1. Galior, K., S. Grebe, and R. Singh, Development of vitamin D toxicity from overcorrection of vitamin D deficiency: a review of case reports. Nutrients, 2018. 10(8): p. 953.

2. Maukonen, J. and M. Saarela, Human gut microbiota: does diet matter? Proceedings of the Nutrition Society, 2015. 74(1): p. 23-36.

3. Masoumi, S.J., et al., The effect of yogurt fortified with Lactobacillus acidophilus and Bifidobacterium sp. probiotic in patients with lactose intolerance. Food Science \& Nutrition, 2021.

4. Kok, C.R. and R. Hutkins, Yogurt and other fermented foods as sources of health-promoting bacteria. Nutrition reviews, 2018. 76(Supplement_1): p. 4-15.

5. Facioni, M.S., et al., Nutritional management of lactose intolerance: the importance of diet and food labelling. Journal of Translational Medicine, 2020. 18(1): p. 1-9.

6. Redondo-Useros, N., et al., Associations of probiotic fermented milk (PFM) and yogurt consumption with Bifidobacterium and Lactobacillus components of the gut microbiota in healthy adults. Nutrients, 2019. 11(3): p. 651.

7. Magne, F., et al., The Firmicutes/Bacteroidetes ratio: a relevant marker of gut dysbiosis in obese patients? Nutrients, 2020. 12(5): p. 1474.

8. Rowland, I., et al., Gut microbiota functions: metabolism of nutrients and other food components. European Journal of Nutrition, 2018. 57(1): p. 1-24.

9. Thursby, E. and N. Juge, Introduction to the human gut microbiota. Biochemical Journal, 2017. 474(11): p. 1823-1836.

10. Flint, H.J., et al., Links between diet, gut microbiota composition and gut metabolism. Proceedings of the Nutrition Society, 2015. 74(1): p. 13-22.

11. Vlasova, A.N., et al., Comparison of probiotic lactobacilli and bifidobacteria effects, immune responses and rotavirus vaccines and infection in different host species. Veterinary immunology and immunopathology, 2016. 172: p. 72-84.

12. Wang, X., et al., Gut microbiota dysbiosis is associated with Henoch-Schönlein Purpura in children. International immunopharmacology, 2018. 58: p. 1-8.

13. Lazar, V., et al., Aspects of gut microbiota and immune system interactions in infectious diseases, immunopathology, and cancer. Frontiers in immunology, 2018. 9: p. 1830.

14. Yue, B., et al., Inflammatory bowel disease: a potential result from the collusion between gut microbiota and mucosal immune system. Microorganisms, 2019. 7(10): p. 440.

15. Lee, A., et al., Consumption of dairy yogurt containing Lactobacillus paracasei ssp. paracasei, Bifidobacterium animalis ssp. lactis and heat-treated Lactobacillus plantarum improves immune function including natural killer cell activity. Nutrients, 2017. 9(6): p. 558. 
16. Shi, N., et al., Interaction between the gut microbiome and mucosal immune system. Military Medical Research, 2017. 4(1): p. 1-7.

17. Segal, B.M., et al., Repeated subcutaneous injections of IL12/23 p40 neutralising antibody, ustekinumab, in patients with relapsing-remitting multiple sclerosis: a phase II, double-blind, placebocontrolled, randomised, dose-ranging study. The Lancet Neurology, 2008. 7(9): p. 796-804.

18. Obrenovich, M.E., Leaky gut, leaky brain? Microorganisms, 2018. 6(4): p. 107.

19. Mackay, F., et al., BAFF AND APRIL: a tutorial on B cell survival. Annual review of immunology, 2003. 21(1): p. 231-264.

20. Ey, P., S.J. Prowse, and C. Jenkin, Isolation of pure IgG1, IgG2a and IgG2b immunoglobulins from mouse serum using protein A-sepharose. immunochemistry, 1978. 15(7): p. 429-436.

21. Waldhauer, I. and A. Steinle, NK cells and cancer immunosurveillance. Oncogene, 2008. 27(45): p. 5932-5943.

22. Trapani, J.A. and M.J. Smyth, Functional significance of the perforin/granzyme cell death pathway. Nature Reviews Immunology, 2002. 2(10): p. 735-747.

23. Lai, Y., et al., Dietary supplementation of Bacillus sp. DU106 activates innate immunity and regulates intestinal microbiota in mice. Journal of Functional Foods, 2020. 75: p. 104247.

24. Thapa, P. and D.L. Farber, The role of the thymus in the immune response. Thoracic surgery clinics, 2019. 29(2): p. 123-131.

25. Majumdar, S., S. Pathak, and D. Nandi, Thymus. Resonance, 2018. 23(2): p. 197-217.

26. Chairatana, P. and E.M. Nolan, Defensins, lectins, mucins, and secretory immunoglobulin A: microbebinding biomolecules that contribute to mucosal immunity in the human gut. Critical reviews in biochemistry and molecular biology, 2017. 52(1): p. 45-56.

27. Joukar, F., et al., Gut microbiota as a positive potential therapeutic factor in carcinogenesis: an overview of microbiota-targeted therapy. Journal of gastrointestinal cancer, 2020. 51(2): p. 363-378. 